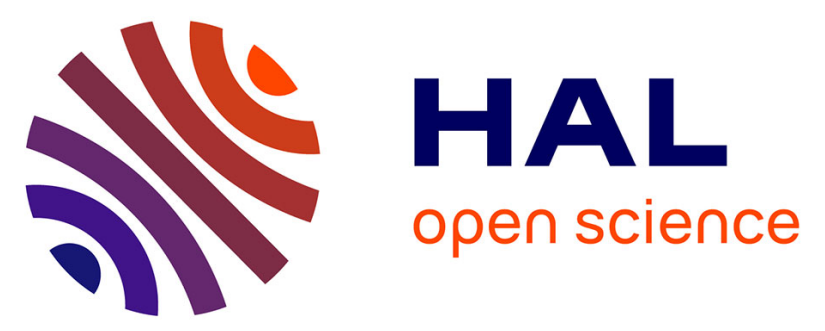

\title{
Évaluation de la contamination par les éléments-traces métalliques dans une zone minière du Maroc oriental
}

Abdelaziz Smouni, Mohammed Ater, Florence Auguy, Laurent Laplaze,

Mohammed El Mzibri, Fatiha Berhada, Abdelkarim Filali-Maltouf, Patrick

Doumas

\section{To cite this version:}

Abdelaziz Smouni, Mohammed Ater, Florence Auguy, Laurent Laplaze, Mohammed El Mzibri, et al.. Évaluation de la contamination par les éléments-traces métalliques dans une zone minière du Maroc oriental. Cahiers Agricultures, 2010, 19 (4), pp.273-279. 10.1684/agr.2010.0413 . hal-01219555

\section{HAL Id: hal-01219555 \\ https://hal.science/hal-01219555}

Submitted on 22 Oct 2015

HAL is a multi-disciplinary open access archive for the deposit and dissemination of scientific research documents, whether they are published or not. The documents may come from teaching and research institutions in France or abroad, or from public or private research centers.
L'archive ouverte pluridisciplinaire HAL, est destinée au dépôt et à la diffusion de documents scientifiques de niveau recherche, publiés ou non, émanant des établissements d'enseignement et de recherche français ou étrangers, des laboratoires publics ou privés.

\section{(c) (1) $\$$}

Distributed under a Creative Commons Attribution - NonCommerciall 4.0 International 


\title{
Évaluation de la contamination par les éléments-traces métalliques dans une zone minière du Maroc oriental ${ }^{*}$
}

\author{
Abdelaziz Smouni $^{1}$ \\ Mohammed Ater ${ }^{2}$ \\ Florence Auguy ${ }^{3}$ \\ Laurent Laplaze ${ }^{3}$ \\ Mohammed El Mzibri \\ Fatiha Berhada ${ }^{4}$ \\ Abdelkarim Filali-Maltouf ${ }^{4}$ \\ Patrick Doumas ${ }^{3}$ \\ ${ }^{1}$ CNESTEN \\ Centre national de l'énergie, \\ des sciences et des techniques nucléaires \\ Laboratoire de biotechnologie des plantes \\ UBRM-DSV \\ BP 1382 R.P \\ 10001 Rabat \\ Maroc \\ <azizsmouni@yahoo.com> \\ <mzribi@yahoo.com> \\ ${ }^{2}$ Faculté des sciences \\ Université Abdelmalek Essaâdi \\ Laboratoire "Diversité et conservation \\ des systèmes biologiques " \\ BP 2062 \\ 93002 Tétouan \\ Maroc \\ <mohammed.ater@gmail.com> \\ ${ }^{3}$ IRD \\ Institut de recherche pour le développement \\ Équipe Rhizogenèse (INRA/IRD/UM2) \\ UMR DIAPC \\ 911, Av. Agropolis \\ 34394 Montpellier cedex 5 \\ France \\ <patrick.doumas@ird.fr> \\ $<$ laurent.laplaze@ird.fr> \\ <florence.auguy@ird.fr> \\ ${ }^{4}$ Faculté des sciences \\ Université Mohammed V - Agdal \\ Laboratoire de microbiologie et biologie \\ moléculaire \\ Av. Ibn Batouta \\ BP 1014 \\ 10001 Rabat \\ Maroc \\ $<$ filalimaltouf@gmail.com> \\ $<$ fatibrhada@hotmail.com>
}

Tirés à part : P. Doumas

\begin{abstract}
Résumé
La contamination des sols et des résidus miniers dans la région orientale du Maroc a été évaluée par dosage des éléments-traces métalliques (ETM) (As, Cd, Cu, Ni, Pb et Zn) par ICP-AES (inductively coupled plasma-atomic emission spectrometry). Ce travail révèle une contamination importante des sites étudiés et souligne une grande variabilité dans les concentrations en ETM en fonction de l'élément analysé et du site étudié. L'index de pollution des stations étudiées est généralement très élevé du fait de la présence simultanée de plusieurs de ces éléments. Cette combinaison de différents ETM rend les sols de la région toxiques pour la flore, la faune et les populations locales. Une analyse des espèces végétales qui se développent sur ces zones polluées est envisagée dans le cadre de la mise en place d'un programme de phytoremédiation.
\end{abstract}

Mots clés : élément métallique ; Maroc ; oligo-élément ; pollution du sol ; résidu.

Thèmes : pathologie ; ressources naturelles et environnement ; sols.

\section{Abstract \\ Assessment of contamination by metallic trace elements in a mining area of eastern Morocco}

Soil and tailings contamination in the eastern region of Morocco was investigated by determining metallic trace elements (MTE) ( $\mathrm{As}, \mathrm{Cd}, \mathrm{Cu}, \mathrm{Ni}, \mathrm{Pb}$ and $\mathrm{Zn}$ ) using inductively coupled plasma-atomic emission spectrometry (ICP-AES). This work revealed extensive contamination of the sites studied and also showed a great variability in the concentrations of MTE according to the element analyzed and the site. The soil index pollution is considered very high because of the simultaneous presence of these elements. This combination of MTE in soils makes the region toxic to flora, fauna and local populations. An exploration of plant species growing on these contaminated areas will be considered in the context of the implementation of a program for phytoremediation.

Key words: metallic elements; Morocco; residues; soil pollution; trace elements.

Subjects: natural resources and environment; pathology; soils.

L e développement mondial de l'industrialisation a entraîné une utilisation massive de plusieurs types de minerais métallifères et une croissance importante des activités minières. Ces activités minières sont fortement polluantes et leurs impacts sur l'environnement et la santé sont à l'échelle de leur importance économique (Jung, 2001; Navarro et al., 2008).
La plupart des polluants miniers sont nocifs pour la faune et la flore des milieux terrestres et aquatiques. En plus des lixiviats acides et des résidus miniers qui provoquent une détérioration des écosystèmes, les éléments-traces métalliques (ETM) sont à l'origine d'effets encore plus néfastes sur les fonctions physiologiques des organismes vivants (Boularbah et al., 2006 ; Lim et al., 2007). À faibles

\footnotetext{
* Pour citer cet article: Smouni A, Ater M, Auguy F, Laplaze L, El Mzibri M, Berhada F, FilaliMaltouf A, Doumas P. Évaluation de la contamination par les éléments-traces métalliques dans
} une zone minière du Maroc oriental. Cah Agric 2010 ; 19 : 273-9. DOI : 10.1684/agr.2010.0413 
doses, des métaux comme le fer, le zinc, le chrome et le cuivre sont nécessaires à la vie. Cependant, à fortes doses, ils sont toxiques et peuvent être à l'origine de complications fonctionnelles importantes et de troubles de santé graves. Par ailleurs, certains métaux comme le plomb, le mercure ou l'arsenic sont très toxiques et sont classés parmi les polluants dits "polluants atmosphériques dangereux" (Lee et Chon, 2003).

Plusieurs études se sont intéressées à la pollution par les ETM des sites miniers et de leurs alentours et à l'impact de ces activités sur l'environnement (Rybicka, 1995; Lee et al., 2001). Ces études ont montré que la pollution par les ETM peut affecter l'écosystème de manière irréversible si des mesures adéquates de protection ne sont pas prises rapidement. En raison des phénomènes d'érosion dans les zones contaminées, les ETM peuvent se retrouver dispersés dans les réseaux fluviaux ou sous forme d'aérosols à plusieurs kilomètres des sites d'origine. Cette contamination diffuse rend le phénomène encore plus dévastateur et difficile à contrôler (Bell et al., 2001 ; Schwartz et al., 2001 ; Passariello et al., 2002).

L'activité minière est l'un des piliers fondamentaux de l'économie marocaine, et le secteur des métaux a connu un développement substantiel depuis 1919. La production marocaine de plomb avait dépassé 170000 tonnes en 1980, représentant 3,5\% de la production mondiale (Wajdini, 1998). Dans ce contexte, les mines du Maroc oriental ont largement contribué à la production nationale de plomb. Cependant, les activités minières ont conduit à une modification profonde du paysage de la région et à sa contamination par les sous-produits des traitements et les déchets rejetés.

L'objectif de ce travail est de réaliser une évaluation préliminaire du taux de pollution des sols par les éléments-traces métalliques au niveau de l'un des principaux sites miniers du Maroc oriental : le district minier de Boubker et Touissit ainsi que la fonderie d'Oued El Heimer.

\section{Matériel et méthode}

\section{Description des sites}

La région d'étude se situe au niveau de la chaîne des horsts, bande montagneuse qui s'étend sur une longueur de 100 km et une largeur de $20-30 \mathrm{~km}$. Cette chaîne est limitée au nord par le couloir OujdaTaourirt, à l'ouest par la plaine de Tafrata, au sud par le vaste domaine des hauts plateaux et à l'est par la frontière maroco-algérienne. Le site d'étude se situe dans la partie orientale de cette chaîne montagneuse, au niveau de la fonderie d'Oued El Heimer et des mines Touissit et Boubker (figure 1).

La fonderie d'Oued El Heimer, à proximité du village du même nom, se trouve à $33 \mathrm{~km}$ au sud-est de la ville d'Oujda. Cette fonderie encore en activité est spécialisée dans le traitement, la fusion et le raffinage du plomb et de l'argent. Depuis 1945, l'unité traite les minerais provenant de l'ensemble des districts miniers du Maroc oriental. À partir de 1980, suite à l'épuisement des sites marocains, la matière première est principalement importée du Mexique, du Brésil, de la Tunisie et de l'Algérie. Cette région est soumise à une forte pollution aérienne due à l'émission de gaz chargés de poussières fines qui polluent les zones avoisinantes, en particulier les zones situées dans la direction des vents dominants (N-NE). Des tentatives de reboisement de la zone avoisinant la fonderie ont été entreprises mais, en raison des fortes émissions de gaz, le massif forestier situé dans l'axe des vents dominants n'a pu résister à cette pollution (figure $2 \mathrm{~A}$, stations $\mathrm{OH} 1$ à $\mathrm{OH} 7$ ). Seuls persistent quelques arbres situés de part et d'autre de cette zone. Les sols sont également soumis à une forte pollution par une accumulation massive de scories au voisinage direct de la fonderie (figure $2 B$, stations $\mathrm{OH} 8$ à OH11). Ces scories sont disposées au bord d'un cours d'eau qui, en période de crues, en emporte une quantité importante sous forme particulaire ou dissoute. Ce lessivage peut constituer une source majeure de contamination des sols et des points d'eau avoisinants.

Les sites de Boubker et de Touissit, situés à une trentaine de kilomètres au sud de la ville d'Oujda, ont respectivement été fermés en 1975 et 2002, suite à l'épuisement des réserves, après une exploitation de près de 40 ans pour Boubker et de 75 ans pour Touissit. La source de pollution dans cette région est principalement constituée par les déchets de lavage formant des digues de sable de grande superficie. Ces sables sont facilement transportés par les vents et les précipitations sous forme particulaire. Il est important de souligner la présence de nombreuses habitations directement au pied des digues (figure 3A). Par ailleurs, certaines de ces digues ont été recouvertes par un substrat (stérile) correspondant aux matériaux résiduels directement issus du minerai extrait lors de l'exploitation de la mine. La station Touil correspond à la plus ancienne digue de la région de Touissit. Cette digue, recouverte depuis une dizaine d'années, a fait l'objet de reboisement par du pin. Les stations Toui2 et Toui3 sont situées respectivement sur une digue non recouverte et une digue recouverte ayant fait l'objet de reboisement par du pin et de l'acacia. La station Touí correspond à la digue la plus récente, en cours de recouvrement. La station Toui5 est située près de l'entrée du puits de la mine de Touissit. Les stations Toui6 et Toui7 sont situées respectivement dans les zones recouvertes et non recouvertes de la plus grande digue de Touissit. Cette digue porte un couvert épars de pin et d'acacia (figure 3B).

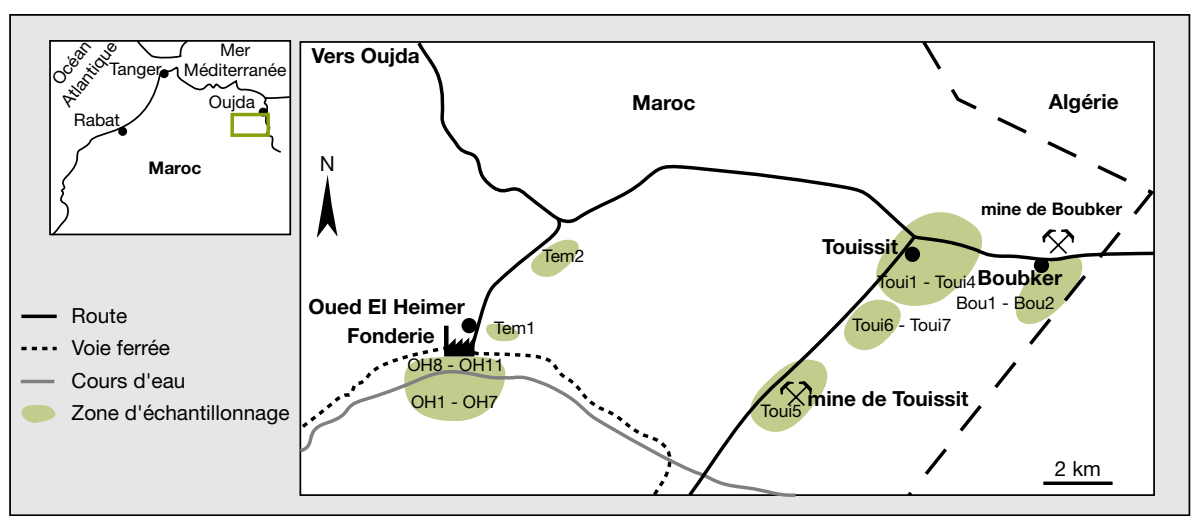

Figure 1. Situation géographique des régions étudiées et localisation des stations de prélèvements.

Figure 1. Geographic settings of the study sites and location of sampling stations. 

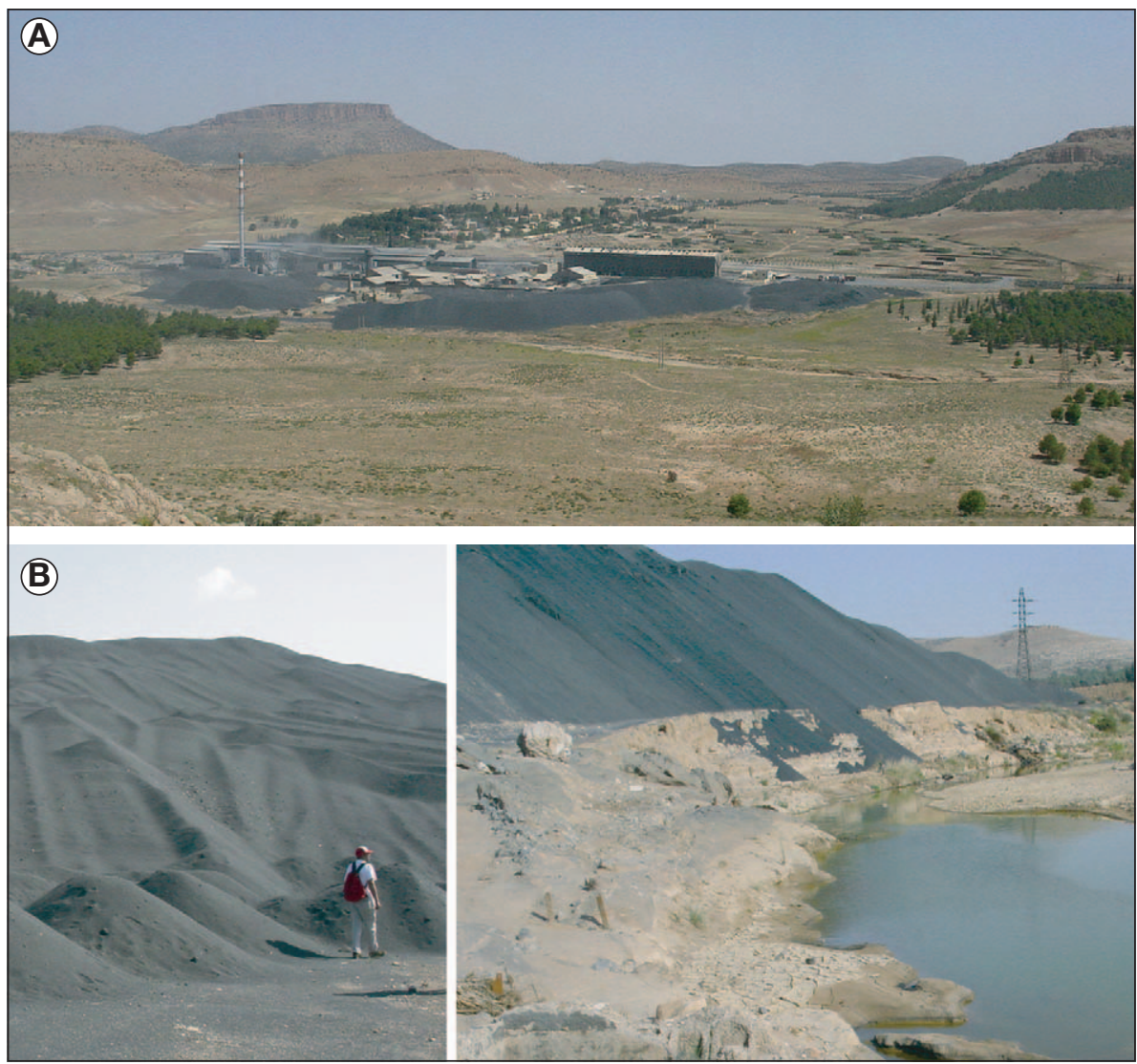

Figure 2. Description du site d'Oued El Heimer.

Figure 2. Description of Oued El Heimer site.

A) vue d'ensemble de la fonderie et de la zone dénudée de végétation située dans le passage des émanations de gaz et de poussière émises par la cheminée; B) scories plombifères déposées sur de grandes surfaces aux abords de la fonderie et du cours d'eau.

Les stations Bou1 et Bou2 sont situées au niveau d'une digue de sable restée nue et sans traitement, ni couverture végétale (figure 3C).

Deux sites témoins - Tem1 et Tem2 - à l'écart des activités minières, ont également été utilisés. Tem1 se situe au niveau du Jbel Magaze, à $1 \mathrm{~km}$ de la fonderie dans le sens opposé au vent dominant et Tem2 entre Touissit et Oued El Heimer. Les échantillons de sol ont été prélevés sur 22 stations dans les trois régions étudiées (tableau 1). Pour l'ensemble des échantillons, la partie superficielle du sol, d'une surface de $0,3 \mathrm{~m}^{2}$, a été prélevée sur $10 \mathrm{~cm}$ de profondeur, homogénéisée et tamisée $(<2 \mathrm{~mm})$.

\section{Mesure du pH}

Dix grammes de sol sont mis en suspension dans $50 \mathrm{~mL}$ d'eau distillée. Le mélange est agité pendant 1 heure à et $\mathrm{Zn}$ sont ensuite déterminées par ICPAES (inductively coupled plasma-atomic emission spectrometry ; Ultima2 JY) selon la méthode de Marguí et al. (2005).

\section{Résultats et discussion}

\section{Mesures du pH}

Les résultats obtenus montrent des valeurs de $\mathrm{pH}$ neutre à faiblement acide, variant entre 5,5 et 7,8 (tableau 2). Parmi l'ensemble des prélèvements, seuls deux prélèvements présentent des $\mathrm{pH}$ légèrement acides dans le site d'Oued El Heimer. Des résultats similaires ont été rapportés par Pichtel et al. (2000) pour des sols avoisinant une fonderie de plomb aux États-Unis. Saïdi (2004) rapporte des valeurs de $\mathrm{pH}$ comprises entre 7,5 et 8,1 dans le district minier de Zaïda dans le Maroc oriental. Cependant, Navarro et al. (2008) ont trouvé des valeurs de $\mathrm{pH}$ comprises entre 3,1 et 8,4 dans les régions minières de Cabezo Rajao en Espagne. Plusieurs auteurs ont montré que le $\mathrm{pH}$ du sol joue un rôle majeur dans la solubilité et la biodisponibilité des métaux (Basta et al., 1993 ; Jung et Thornton, 1996). Dans notre étude, de nombreux sites présentent des $\mathrm{pH}$ alcalins dont l'origine résulte généralement de la présence de carbonates dans le sol. Ces carbonates peuvent être un réservoir important pour les ETM du sol. KabataPendias et Pendias (1992) rapportent que le $\mathrm{Cd}$, le $\mathrm{Cu}$, le $\mathrm{Pb}$ et le $\mathrm{Zn}$ ont une affinité particulièrement élevée pour les carbonates. Ainsi, dans les milieux où les concentrations en plomb sont relativement élevées, les carbonates sous forme d'hydrocérusite et de cérusite, sont les régulateurs majeurs de la distribution de cet élément dans les sols et les eaux superficielles (Essington et al., 2004).

\section{Mesure de la concentration en ETM dans les sols}

Les concentrations en $\mathrm{As}, \mathrm{Cd}, \mathrm{Cu}, \mathrm{Ni}$, $\mathrm{Pb}$ et Zn dans les échantillons de sol prélevés dans les différentes stations sont présentées dans le tableau 2.

Les régions minières de Touissit et Boubker se caractérisent par des teneurs qui varient entre 11 et $187 \mathrm{ppm}$ d'As, entre 0 et 57,5 ppm de Cd, entre 31 et $2253 \mathrm{ppm}$ de $\mathrm{Cu}$, entre 0 et 12,3 ppm de $\mathrm{Ni}$, entre 84 et $7458 \mathrm{ppm}$ de $\mathrm{Pb}$ et 


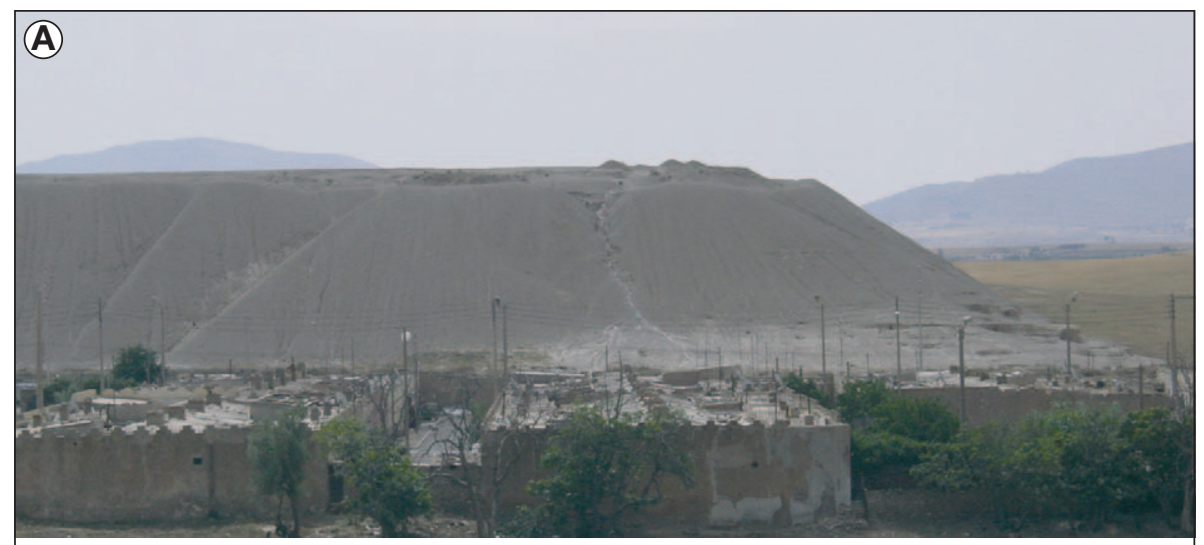

(B)
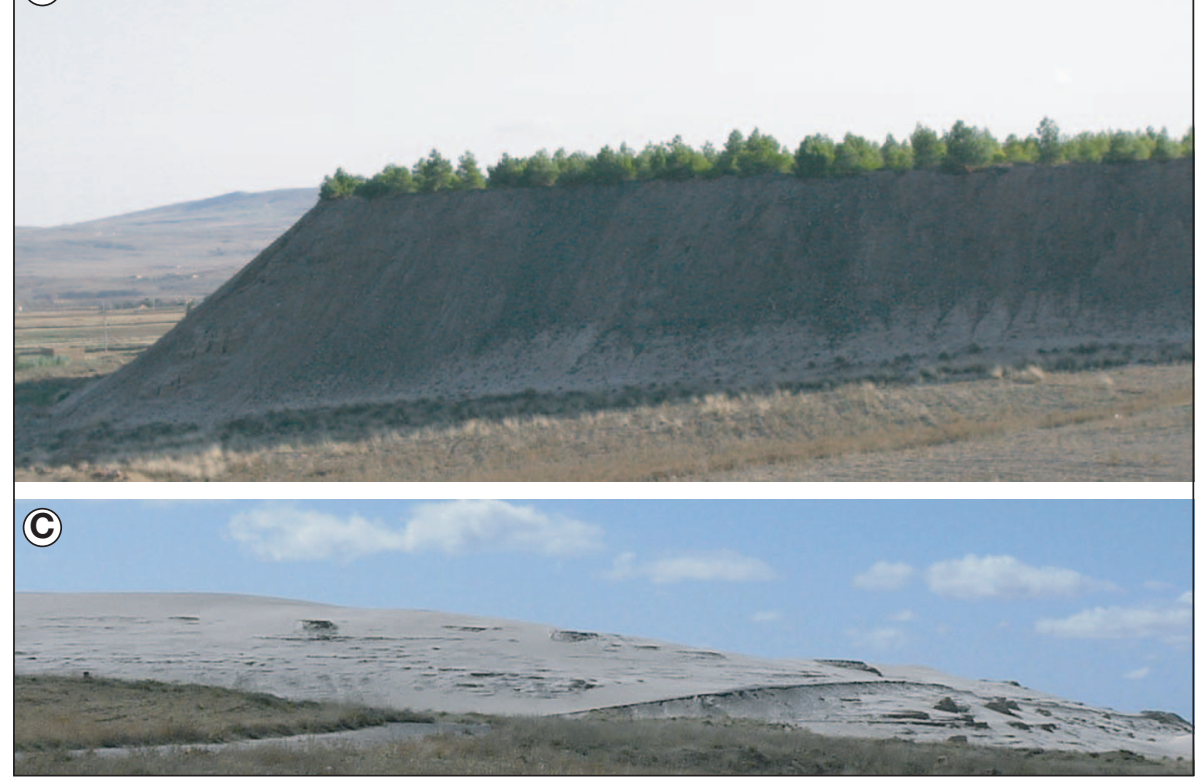

Figure 3. Sites de Touissit et Boubker.

Figure 3. Touissit and Boubker sites.

A) digue de sable aux abords du village de Touissit ; B) reboisement avec de I'Acacia et du Pin au niveau d'une digue recouverte par du stérile ; C) digue de sable à proximité d'un champ de blé dans la région de Boubker.

entre 171 et $2256 \mathrm{ppm}$ pour le $\mathrm{Zn}$. Les teneurs en As, Cd et Cu sont comparables à celles trouvées dans la région de Cabezo Rajao en Espagne par Navarro et al. (2008). En revanche, les teneurs en $\mathrm{Pb}$ et $\mathrm{Zn}$ sont nettement supérieures à celles rapportées par ces auteurs.

La région d'Oued El Heimer, où se situe la fonderie, présente des teneurs en As situées entre 7 et 411 ppm, en Cd entre 0,1 et $164,5 \mathrm{ppm}$, en $\mathrm{Cu}$ entre 0,3 et 531,6 ppm et en $\mathrm{Ni}$ entre 0,1 et 34,4 ppm. Cette région se caractérise par de fortes teneurs en $\mathrm{Zn}$ et $\mathrm{Pb}$ en particulier au niveau des scories. Ces valeurs varient entre 10 et $26776 \mathrm{ppm}$ pour le $\mathrm{Zn}$ et entre 26 et $9478 \mathrm{ppm}$ pour le $\mathrm{Pb}$. Bien que ces valeurs semblent élevées,
La variation des concentrations en ETM entre les sites et même entre les différentes zones d'un même site semble être une caractéristique de la pollution des sites miniers. Ernst et Nelissen (2000) ont montré que les concentrations en métaux totaux dans les sols orogéniques, ainsi que dans les sols proches des fonderies, sont très élevées mais très variables d'un site à l'autre.

Les échantillons récoltés aux abords de la fonderie d'Oued El Heimer, et en particulier au niveau des scories, présentent des concentrations plus élevées en As, Cd, Pb et $\mathrm{Zn}$ que dans les deux régions minières décrites. Les teneurs en $\mathrm{Cu}$ et $\mathrm{Ni}$ sont également importantes. De nombreux auteurs ont montré que les régions à proximité des fonderies sont extrêmement polluées par les émissions de particules riches en ETM durant le processus de fusion et leur accumulation subséquente à la surface du sol (Li et Thornton, 1993 ; Bloemen et al., 1995 ; Dudka et al., 1996). Les sols des sites de fonderies causent plus de problèmes environnementaux que les sites d'extraction, du fait que, dans ces zones, les métaux sont plus mobiles et biodisponibles (Li et Thornton, 1993).

Dans les sols d'une mine de $\mathrm{Pb}$ et $\mathrm{Zn}$ en Corée du Sud, des concentrations en Zn variant entre 329 et 25800 ppm ont été trouvées (Jung et Thornton, 1996). Ces valeurs rentrent dans l'intervalle de variation des résidus de la région étudiée. Par ailleurs, Li et Thornton (1993) ont rapporté des concentrations en Zn atteignant 45900 ppm dans la région minière de Shipham en Grande-Bretagne. Dans le district minier de Zaïda, Saïdi (2004) rapporte des concentrations en Zn allant jusqu'à 409741 ppm et Boularbah et al. (2006) présente des valeurs en $\mathrm{Zn}$ de 108000 ppm dans les résidus d'une mine polymétallique de la région de Guemassa au Maroc. Ces valeurs sont plus élevées que celles trouvées dans les sites étudiés. Les concentrations en $\mathrm{Pb}$ dans notre site d'étude sont normalement élevées puisqu'il s'agit de mines et de sites d'exploitation dédiés au $\mathrm{Pb}$, au $\mathrm{Zn}$ et à l'Ag. Dans d'autres études, plusieurs équipes ont trouvé des teneurs plus importantes en $\mathrm{Pb}$ dans les déchets miniers. Li et Thornton (1993) ont rapporté des concentrations en $\mathrm{Pb}$ variant de 3280 à 28900 ppm dans une région minière de $\mathrm{Pb}$ et $\mathrm{Zn}$, en GrandeBretagne. Saïdi (2004) et Boularbah et al. (2006) rapportent respectivement des valeurs de 73936 et 30100 ppm de $\mathrm{Pb}$ dans des résidus de fonderies situées dans l'Atlas marocain. 
Tableau 1. Description des échantillons analysés.

Table 1. Description of analyzed samples.

\begin{tabular}{|c|c|c|c|c|}
\hline Site & Code & pH & Coordonnées GPS & Nature de l'échantillon \\
\hline \multirow[t]{2}{*}{ Témoin } & Tem1 & 7,7 & alt : $995 \mathrm{~m} \mathrm{~N} 34^{\circ} 2730^{\prime} \mathrm{W} 001^{\circ} 53$ 46' & Sol végétalisé \\
\hline & Tem2 & 7,7 & alt : $1134 \mathrm{~m} \mathrm{~N} 34^{\circ} 2815^{\prime} \mathrm{W} 001^{\circ} 4634^{\prime}$ & Sol végétalisé \\
\hline \multirow{11}{*}{$\begin{array}{l}\text { Oued } \\
\text { El Heimer }\end{array}$} & $\mathrm{OH} 1$ & 7,3 & alt : $981 \mathrm{~m} \mathrm{~N} 34^{\circ} 265^{\prime \prime} \mathrm{W} 001^{\circ} 53$ 97' & Sol végétalisé \\
\hline & $\mathrm{OH} 2$ & 6,7 & alt : 981 m N 34² 26 59' W 00153 97' & Sol végétalisé \\
\hline & $\mathrm{OH} 3$ & 7,1 & alt : $981 \mathrm{~m} \mathrm{~N} 34^{\circ} 265^{\prime} \mathrm{W} 001^{\circ} 53$ 97' & Sol végétalisé \\
\hline & $\mathrm{OH} 4$ & 7,0 & alt : 981 m N $34^{\circ} 26$ 59' W 00153 97' & Sol végétalisé \\
\hline & $\mathrm{OH} 5$ & 7,6 & alt : 981 m N 34² 26 59' W $001^{\circ} 53$ 97' & Sol végétalisé \\
\hline & $\mathrm{OH} 6$ & 5,5 & 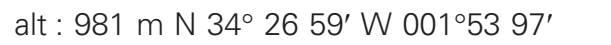 & Sol végétalisé \\
\hline & $\mathrm{OH} 7$ & 7,7 & alt : 981 m N 34 $265^{\circ} 9^{\prime} \mathrm{W} 001^{\circ} 53$ 98' & Sol végétalisé sous pinède \\
\hline & $\mathrm{OH} 8$ & 7,1 & 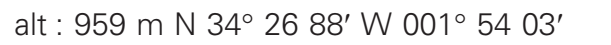 & Scories issues de la fonderie \\
\hline & $\mathrm{OH} 9$ & 6,1 & 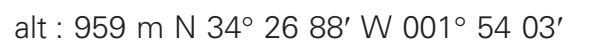 & Scories issues de la fonderie \\
\hline & $\mathrm{OH} 10$ & 7,9 & 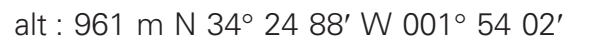 & Scories issues de la fonderie \\
\hline & $\mathrm{OH} 11$ & 7,2 & 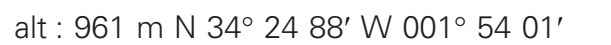 & Scories issues de la fonderie \\
\hline \multirow[t]{7}{*}{ Touissit } & Toui1 & 7,7 & alt : $1132 \mathrm{~m} \mathrm{~N} 34^{\circ} 2826^{\prime} \mathrm{W} 001^{\circ} 46$ 19' $^{\prime}$ & Stérile recouvrant la digue \\
\hline & Toui2 & 7,6 & alt : $1134 \mathrm{~m} \mathrm{~N} 34^{\circ} 2815^{\prime} \mathrm{W} 001^{\circ} 4634^{\prime}$ & Digue de sable (résidus de traitement du minerai) \\
\hline & Toui3 & 7,2 & alt : $1134 \mathrm{~m} \mathrm{~N} 34^{\circ} 2815^{\prime} \mathrm{W} 001^{\circ} 4634^{\prime}$ & Stérile recouvrant la digue \\
\hline & Toui4 & 7,2 & 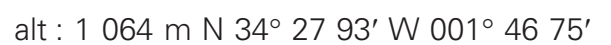 & Stérile recouvrant la digue \\
\hline & Toui5 & 7,4 & alt : $1055 \mathrm{~m} \mathrm{~N} 34^{\circ} 2649^{\prime} \mathrm{W} 001^{\circ} 48$ 22' & Stérile \\
\hline & Toui6 & 6,9 & 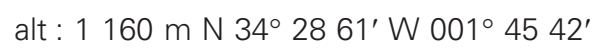 & Digue de sable (résidus de traitement du minerai) \\
\hline & Toui7 & 6,8 & alt : $1160 \mathrm{~m} \mathrm{~N} 34^{\circ} 2861^{\prime} \mathrm{W} 001^{\circ} 4542^{\prime}$ & Stérile recouvrant la digue \\
\hline \multirow[t]{2}{*}{ Boubker } & Bou1 & 7,7 & 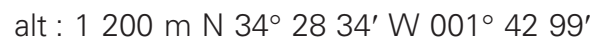 & Digue de sable (résidus de traitement du minerai) \\
\hline & Bou2 & 7,8 & alt : $1189 \mathrm{~m} \mathrm{~N} 34^{\circ} 2831^{\prime} \mathrm{W} 001^{\circ} 432^{\prime \prime}$ & Digue de sable (résidus de traitement du minerai) \\
\hline
\end{tabular}

Pour le Cu, Bennisse et al. (2005) rapportent des teneurs atteignant 424 ppm dans la région minière de Draâ Sfar au Maroc. Cette valeur est comparable à la concentration trouvée dans les scories d'Oued El Heimer. Par ailleurs, des concentrations atteignant 1400 et 1899 ppm ont été trouvées respectivement près du site minier de Sambo en Corée du Sud (Jung et Thornton, 1996) et dans les résidus de fonderies du district minier de Zaïda (Saïdi, 2004).

Les travaux de Navarro et al. (2008) ont mis en évidence des concentrations en Cd atteignant 332 ppm dans les sols prélevés à partir d'une ancienne région minière de $\mathrm{Pb}-\mathrm{Zn}$ en Espagne. Fuge et al. (1993) ont trouvé des valeurs de 980 ppm de Cd dans des sols près des mines métallifères au Pays de Galles. Saïdi (2004) rapporte des concentrations en Cd de 1046 ppm dans le site minier de Mibladen au Maroc. Ces valeurs sont nettement plus élevées que celles trouvées dans les régions d'Oued El Heimer, Touissit et Boubker. Cependant, certains auteurs ont trouvé des valeurs en Cd proches de nos résultats. Ainsi, Boularbah et al. (2006) et
Lim et al. (2007) rapportent respectivement des concentrations de 228 ppm et de 20 ppm en Cd.

Dans notre étude, excepté pour le Ni, la concentration en ETM dans les sols témoins est inférieure ou proche de la moyenne mondiale des sols non contaminés (tableau 2, VN). En revanche, la majorité des sols issus des zones qui présentent une activité minière ont des concentrations élevées en un ou plusieurs de ces éléments. Ces concentrations élevées en EMT sont donc directement liées aux activités minières qui durent depuis des décennies dans la région. Des résultats similaires ont été trouvés par de nombreux auteurs (Matthews et Thornton, 1982; Saidi, 2004).

\section{Index de pollution par les ETM dans les sols}

La concentration critique du sol est définie comme étant la valeur au-dessus de laquelle la toxicité est possible. Ces valeurs sont de 8 ppm pour le $\mathrm{Cd}$, $125 \mathrm{ppm}$ pour le $\mathrm{Cu}$ et $400 \mathrm{ppm}$ pour le $\mathrm{Pb}$ et le $\mathrm{Zn}$ (Kabata-Pendias et Pendias,
1992). Dans notre étude, les résultats obtenus montrent que les concentrations en métaux sont au-dessus du niveau critique dans la plupart des stations. Quatrevingts pour cent des stations présentent des valeurs supérieures au seuil critique pour au moins trois de ces éléments. Les scories d'Oued El Heimer et les digues de Touissit et Boubker sont particulièrement riches en ces quatre éléments. Ces sols sont également riches en As. Néanmoins, la toxicité de cet élément est difficile à apprécier dans le sol puisqu'elle dépend de sa biodisponibilité, généralement très faible ${ }^{1}$.

La pollution par les métaux dans les sites miniers est généralement associée à une contamination multiple qui augmente considérablement le degré de toxicité des sols (Nimick et Moor, 1991 ; Chon et al., 1998). L'index de pollution (IP) est un critère permettant d'évaluer la toxicité d'un sol. Il permet d'identifier une contamination de type multiéléments dans les échantillons (Chon et al., 1998). Cet index

\footnotetext{
Conseil canadien des ministres de l'environnement Extrait de la publication 1999 ; 1300; ISBN 1-896997-36-8.
} 
Tableau 2. Concentration en éléments-traces métalliques (ETM) ( $\mathrm{As}, \mathrm{Cd}, \mathrm{Cu}, \mathrm{Ni}, \mathrm{Pb}$ et $\mathrm{Zn}$ ) (mg/kg) et indice de pollution (IP) dans les échantillons.

Table 2. Amount of metallic trace elements (MTE) $(\mathrm{mg} / \mathrm{kg})$ and pollution index (IP) in samples.

\begin{tabular}{llrrrrrrr}
\hline Site & Station & As & Cd & Cu & Ni & Pb & Zn & IP \\
\hline Témoin & Tem1 & 37 & nd & 15 & 26 & 286 & 106 & 0,84 \\
Oued El Heimer & Tem2 & nd & nd & 7 & 21 & 24 & 9 & 0,08 \\
& OH1 & 21 & nd & 86 & 34 & 4685 & 781 & 12,58 \\
& OH2 & 7 & 9 & 10 & 1 & 725 & 96 & 2,66 \\
& OH3 & 16 & 1 & 4 & 2 & 53 & 302 & 0,43 \\
& OH4 & 10 & 1 & 1 & nd & 26 & 527 & 0,55 \\
& OH5 & 16 & 2 & 3 & nd & 58 & 10 & 0,37 \\
& OH6 & 16 & 4 & 8 & 1 & 125 & 1146 & 1,60 \\
& OH7 & 30 & 12 & 67 & 2 & 4174 & 2231 & 11,82 \\
& OH8 & 249 & 7 & 134 & 6 & 7179 & 17786 & 33,73 \\
Touissit & OH9 & 201 & 17 & 336 & 6 & 7407 & 11210 & 30,09 \\
& OH10 & 411 & 5 & 509 & 26 & 9479 & 26776 & 47,69 \\
& OH11 & 331 & 165 & 533 & 15 & 4562 & 886 & 27,18 \\
& Toui1 & 11 & 4 & 100 & nd & 1212 & 171 & 3,80 \\
& Toui2 & 14 & 3 & 32 & nd & 264 & 206 & 1,19 \\
& Toui3 & 13 & nd & 223 & nd & 7458 & 725 & 19,81 \\
Boubker & Toui4 & 47 & 15 & 766 & 3 & 1817 & 315 & 7,96 \\
VN & Toui5 & 101 & 11 & 643 & 9 & 3 083 & 252 & 10,48 \\
& Toui6 & 187 & 18 & 2243 & 3 & 3309 & 472 & 15,78 \\
\hline
\end{tabular}

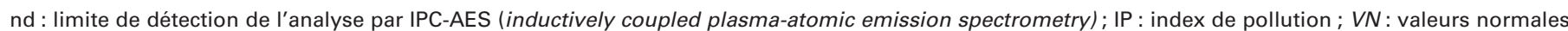
selon Bowen (1979).

est calculé par le rapport des concentrations des métaux dans le sol sur la base de valeurs ajustées correspondant aux niveaux tolérables des concentrations en métaux dans le sol $(\mathrm{IP}=(\mathrm{Cd} / 3+\mathrm{Cu} / 100+$ $\mathrm{Pb} / 100+\mathrm{Zn} / 300) / 4)$. Ainsi, un IP supérieur à 1 correspond à un sol pollué.

Dans le cas de cette étude, l'index de pollution a été calculé pour les différents sites de prélèvement et les résultats sont reportés dans le tableau 1. Pour les stations où la concentration en Cd est inférieure au seuil de détection, la valeur est considérée comme nulle.

Les résultats obtenus montrent des valeurs d'IP très variables d'une station à une autre. Ces valeurs sont comprises entre 0,43 et 47,69. Les indices les plus bas sont obtenus sur les sols témoins et quelques sols végétalisés d'Oued El Heimer. En outre, sur les 22 échantillons testés, 17 ont des IP supérieurs à 1, confirmant la forte contamination de notre site d'étude par les ETM. Des valeurs similaires sont fréquemment observées dans les régions qui présentent des activités minières. Jung (2001) et Lee et al. (2001) rapportent des valeurs d'IP supérieures à 1 dans les sols de plusieurs régions minières en Corée. Saïdi (2004) présente des valeurs d'IP atteignant 14 dans les mines de Zaïda. Bien que dans la région étudiée les concentrations en $\mathrm{Pb}$ et $\mathrm{Zn}$ soient inférieures aux sols de Zaïda, les IP sont, pour certains échantillons, nettement supérieurs à ceux trouvés par Saïdi (2004). Sur les 20 sites pollués, $85 \%$ des échantillons analysés ont un IP supérieur à 1 , et $45 \%$ sont très fortement pollués avec un IP supérieur à 10. Les IP les plus élevés sont enregistrés au niveau des scories aux abords de la fonderie, soulignant le caractère extrêmement toxique de ces milieux. Une analyse statistique non paramétrique (test de Wilcoxon, Statitica 7) révèle une très forte corrélation entre la valeur d'IP et les teneurs en $\mathrm{Pb}$ et $\mathrm{Zn}$ dans les milieux étudiés, et, dans une moindre mesure, avec l'As, donnant un poids particulier à ces trois éléments dans la toxicité des substrats. Ces résultats montrent que la combinaison de métaux présents dans les sols de la région de Touissit, Boubker et Oued El Heimer est extrêmement toxique. Ces ETM fournissent une source de dispersion continue et peuvent être à l'origine d'une pollution endémique de la région.

\section{Conclusion}

L'analyse de la composition en métaux des sols (sols végétalisés, digues, stériles et scories) des sites miniers de Touissit et Boubker et de la fonderie d'Oued El Heimer a révélé des taux élevés en ETM. L'index de pollution, souvent très élevé, montre que la combinaison en métaux dans les sols de la région est une source potentielle de toxicité pour la flore, la faune et les habitants. En plus des activités minières, ces régions connaissent une activité agricole vivrière à prendre en 
considération. On y dénombre plusieurs cultures céréalières ainsi que des plantations d'arbres fruitiers (oliviers, pruniers et pommiers). L'activité pastorale est également omniprésente autour des agglomérations. Ces activités constituent une voie d'entrée des ETM dans la chaîne alimentaire et augmentent ainsi le risque de contamination de la population.

Malgré l'environnement hostile du milieu, plusieurs essences végétales ont été observées sur les sites et sont parfaitement adaptées aux conditions édaphoclimatiques de la région. Ces espèces dites métallicoles participent à la fixation des sols et minimisent ainsi la dissémination des ETM sous l'effet de l'érosion. Certaines de ces plantes ont mis en place des processus adaptatifs leur permettant de tolérer de fortes teneurs en ETM dans le sol. Parmi celles-ci, on peut distinguer les plantes accumulatrices de métaux. Ces plantes présentent un potentiel important pour le développement de stratégies de réhabilitation des sites miniers par des approches de phytoremédiation.

\section{Références}

Baker AJM, Reeves RD, Hajar ASM. Heavy metal accumulation and tolerance in British populations of the metallophyte Thlaspi caerulescens J. \& C. Presl (Brassicaceae). New Phytol 1994; $127: 61-8$.

Basta NT, Pantone DJ, Tabatabai MA. Path analysis of heavy metal adsorption by soil. Agron $J$ $1993 ; 85: 1054-7$.

Bell FG, Bullock SET, Hälbich TFJ, Lindsay P. Environmental impacts associated with an abandoned mine in the Witbank Coalfield, South Africa. Int J Coal Geol 2001 ; 45 : 195-216.
Bennisse R, Labat M, Elasli A, et al. Rhizosphere bacterial populations of metallophyte plants in heavy metal-contaminated soils from mining areas in semiarid climate. World J Microb Biot $2005 ; 20: 759-66$.

Bloemen $\mathrm{ML}$, Markert $\mathrm{B}$, Lieth $\mathrm{H}$. The distribution of $\mathrm{Cd}, \mathrm{Cu}, \mathrm{Pb}$ and $\mathrm{Zn}$ in topsoils of Osnabrück in relation to land use. Sci Tot Environ 1995; 166 137-48.

Boularbah A, Schwartz C, Bitton G, Morel JL. Heavy metal contamination from mining sites in South Morocco: 1. Use of a biotest to assess metal toxicity of tailings and soils. Chemosphere $2006 ; 63: 802-10$

Bowen HYM. Environmental chemistry of element. London: Academic Press, 1979.

Chon HT, Ahn JS, Jung MC. Seasonal variations and chemical forms of heavy metals in soils and dusts from the satellite cities of Seoul. Environ Geochem HIth $1998 ; 20$ : 77-86.

Dudka S, Piotrowska M, Terelak H. Transfer of cadmium, lead and zinc from industrially contaminated soil to crop plants: a field study. Environ Pollut $1996 ; 94$ : 181-8.

Ernst WHO, Nelissen HJM. Life-phase of a zinc- and cadmium-resistant ecotype of Silene vulgaris in risk assessment of polymetallic mine soils. Environ Pollut 2000 ; 107 : 329-38.

Essington ME, Foss JE, Roh Y. The soil mineralogy of lead at Horace' Villa soil. Sci Sol Am J $2004 ; 68: 979-93$

Fuge R, Pearce FM, Pearce NJG, Perkins WT. Geochemistry of $\mathrm{Cd}$ in the secondary environment near abandoned metalliferous mines Wales. Appl Geochem 1993 ; 2 : 29-35.

Jung MC, Thornton I. Heavy metal contamination of soils and plants in the vicinity of a leadzinc mine, Korea. Appl Geochem 1996; 11 : 53-9.

Jung MC. Heavy metal contamination of soils and waters in and around the Imcheon $\mathrm{Au}-\mathrm{Ag}$ mine, Korea. Appl Geochem 2001 ; 16 : 1369-75.

Kabata-Pendias A, Pendias H. Trace elements in soils and plants. Boca Raton (Floride, ÉtatsUnis): CRC Press, 1992

Lee CG, Chon HT, Jung MC. Heavy metal contamination in the vicinity of the Daduk AuAg-Pb-Zn mine in Korea. Appl Geochem 2001 $16: 1377-86$

Lee JS, Chon HT. Exposure assessment of heavy metals on abandoned metal mine areas by ingestion of soil, crop plant and groundwater. J Phys IV France 2003; $107: 757$.
Li X, Thornton I. Multi-element contamination of soils and plants in old mining areas, U.K. Appl Geochem $1993 ; 2$ : 51-6.

Lim HS, Lee JS, Chon HT, Sager M. Heavy metal contamination and health risk assessment in the vicinity of the abandoned Songcheon $\mathrm{Au}-\mathrm{Ag}$ mine in Korea. J Geochem Explor 2007; 96: 223-30.

Marguí $\mathrm{E}$, Queralt I Carvalho $\mathrm{ML}$, Hidalgo $\mathrm{M}$. Comparison of EDXRF and ICP-OES after microwave digestion for element determination in plant specimens from an abandoned mining area. Anal Chim Acta 2005 ; 549 : 197-204.

Matthews HJ, Thornton AK. Seasons and species variation in the content of cadmium and associated metals in pasture at Shipham. Plant Soil $1982 ; 66: 191-3$

Moreno JL, Garcia C, Hernandez T, Pascual JA. Transference of heavy metals from a calcareous soil amended with sewage-sludge compost to barley plants. Bioresource Technol 1996; 55 : 251-8.

Navarro MC, Pérez-Sirvent C, Martínez-Sánchez MJ, Vidal J, Tovar PJ, Bech J. Abandoned mine sites as a source of contamination by heavy metals: A case study in a semi-arid zone. $J$ Geochem Explor 2008 ; 96 : 183-93.

Nimick DA, Moor JM. Prediction of water-soluble metal concentrations in fluvially deposited tailings sediments, Clark Fork Valley, Montana, USA. Appl Geochem 1991 ; 6 : 635-46.

Passariello B, Giuliano V, Quaresima $\mathrm{S}$, et al. Evaluation of the environmental contamination at an abandoned mining site. Microchem $J$ $2002 ; 73: 245-50$.

Pichtel J, Kuroiwa K, Sawyerr HT. Distribution of $\mathrm{Pb}, \mathrm{Cd}$ and $\mathrm{Ba}$ in soils and plants of two contaminated sites. Environ Pollut 2000 ; 110 : 171-8.

Rybicka EH, Jedrzejczyk B. Preliminary studies on mobilisation of copper and lead from contaminated soils and readsorption on competing sorbents. Appl Clay Sci 1995 ; 10 : 259-68.

Saïdi N. Le bassin-versant de la Moulouya: pollution par les métaux lourds et essais de phytoremédiation. Thèse d'Etat, université Mohammed V, Rabat (Maroc), 2004.

Schwartz C, Gerard E, Perronnet K, Morel JL. Measurement of in situ phytoextraction of zinc by spontaneous metallophytes growing on a smelter site. Sci Tot Environ 2001 ; 279 : 215-21.

Wajdini A. Le plomb au Maroc: cas des districts de Touissit et Jbel Aouam. Chronique Recherche Minière 1998 ; 532 : 9-28. 